Voix et Images

voixetimages

\title{
Scénario et fiction : Neige noire
}

\section{Christiane Houde}

Volume 2, numéro 3, avril 1977

Jean Éthier-Blais

URI : https://id.erudit.org/iderudit/200074ar

DOI : https://doi.org/10.7202/200074ar

Aller au sommaire du numéro

\section{Éditeur(s)}

Les Presses de l'Université du Québec

\section{ISSN}

0318-9201 (imprimé)

1705-933X (numérique)

Découvrir la revue

\section{Citer cet article}

Houde, C. (1977). Scénario et fiction : Neige noire. Voix et Images, 2(3), 418-435.

https://doi.org/10.7202/200074ar d'utilisation que vous pouvez consulter en ligne.

https://apropos.erudit.org/fr/usagers/politique-dutilisation/ 


\section{Scénario et fiction : Neige noire}

Jusqu'où peut-on aller dans la contestation des lois de la fiction, tout en restant dans le cadre romanesque? C'est la question qu'on peut se poser a la lecture de Neige noire'. D'abord le titre.

Contrairement aux titres des précédents romans, celui-ci acquiert sa signification non pas dans une analyse sémantique, mais dans un renversement phonétique. $\mathrm{j} \varepsilon_{3} \mathrm{j} w \mathrm{ar}_{\mathrm{r}}$ devient $\mathrm{j} \mathrm{w}(\mathrm{a}) \mathrm{r} \mathrm{j} \varepsilon \mathbf{3}$ et par un glissement phonétique assez facile $\mathrm{O}_{\mathrm{r}} \mathrm{r} \varepsilon \mathbf{3}$, cette Norvège où se situe la majeure partie de ce livre qui porte en sous-titre l'appellation de roman. II y a aussi une tension entre la qualité principale de neige blanche - et son qualificatif ici attribué: noire ${ }^{2}$.

Le roman se présente d'un seul bloc, sans aucune division. Les précédents romans, même s'ils ne comportaient pas de numérotation, se laissaient diviser en chapitres à cause des pages blanches qui ponctuaient le texte. Ici, il n'y a aucune page blanche, aucune division possible. Des paragraphes se suivent où l'on a un scénario-présentation des lieux, des personnages, des situations, et des indications de plans, de caméra c'est un texte continu. On a ensuite les répliques échangées par les personnages. Puis, en dernier lieu, on a les parenthèses - ou crochets: c'est le seul élément typographique nettement délimité. C'est là le lieu des réflexions sur le scénario, sur le temps, sur les réactions des spectateurs. C'est à l'intérieur de ces parenthèses que se devine la présence d'un narrateur qui ne se découvrira - seule apparition du je - que dans les derniers mots du roman:

(...Le temps me dévore, mais de sa bouche, je tire mes histoires, de sa sédimentation mystérieuse, je tire ma semence d'éternité. Eva et Linda approchent de ce thétre illuminé où la pièce qu'on représente est une parabole dans laquelle toutes les œuvres humaines sont enchâssées.) (NN, p. 254) (Ici et ailleurs, les soulignés sont de nous.)

Ce n'est vraiment qu'à la fin qu'on peut rétablir le déroulement chronologique de l'intrigue. Le récit est présenté par fragments avec beaucoup de flashes back et de projections. L'analogie suscite beaucoup de rapprochements visuels ou même de superpositions de scènes, d'images. 
Dans l'œuvre de Hubert Aquin, la mise en abîme est fréquente: il y a toujours un roman dans le roman. Dans l'Antiphonaire, l'auteur écrit un livre dans lequel Christine écrit un roman où il est question de Chigi qui publie et réécrit l'œuvre de Beausang. Dans Trou de mémoire, le roman est écrit par quatre narrateurs. Dans Prochain Épisode, un narrateur écrit lui aussi un roman. Dans Neige noire, ce n'est plus le román dans le roman, mais le scénario dans le scénario. A un moment du livre, la distance tend vers zéro entre le narrateur de Neige noire et Nicolas, personnage-comédien qui devient scénariste. Ce rapprochement se fait surtout des pages 140 à 246, où Nicolas écrit son scénario dans son cahier noir résurgence de P.X. Magnant? Mais à la page 240, la distance se rétablit entre Nicolas et le narrateur. On se retrouve dans l'appartement de Linda et Nicolas est parti à Repulse Bay pour tourner son film.

L'espace géographique est très important dans les romans d'Aquin. Dans Prochain Épisode, on a Genève-lac Léman / Montréal-Cantons de l'Est. Dans Trou de mémoire, on a Montréal-Londres-Montréal / LagosLausanne-Montréal. Dans l'Antiphonaire, on a San Diego-Montréal-Cantons de l'Est3-Montréal / Turin-Genève-Lyon. Dans Neige noire, Montréal est le lien évident d'un axe nord-sud Montréal-Spitzberg-Montréal / MontréalNatchez-under-the-Hill-Montréal4. Les deux pôles Spitzberg et Natchez contiennent chacun leur secret, leur mystère. Spitzberg est la conséquence de Natchez; on ne connaît cette dernière ville, que par la gravure suspendue au-dessus du lit de Michel Lewandowski, dans son appartement de Montréal.

Neige noire se prête très mal à l'exercice du traditionnel résumé de l'œuvre. On peut se reporter à la parenthèse où le narrateur récapitule lui-même le scénario, en pages 243 et 244 .

Cette fois, c'est la réalité qui est contaminée par une fiction encore plus frissonnante. Sylvie Dubuque s'est substituée à Sylvie Lewandowski comme le pareil au même, mais voilà que Sylvie retrouve son identité nominale dans les bras de son père et sous le regard du spectateur. Nicolas Vanesse, jaloux de cet amant qu'elle a gardé, vu et revu, découvre que cet homme est Michel Lewandowski, père de Sylvie. Les deux époux entreprennent leur voyage de noces en Norvège et dans l'archipel du Svalbard, masqués l'un à l'autre: Sylvie n'est pas Sylvie et Nicolas connaît son inavouable secret. (NN, p. 243244)

Quand le roman commence, Nicolas et Sylvie vivent ensemble à Montréal. Nicolas, comédien à Montréal, et Sylvie partent en voyage de noces vers Spitzberg. Nicolas avoue à Sylvie, au moment du départ, qu'il change de métier pour faire du cinéma. On suit le couple tout au long du voyage. Sylvie disparaît lors d'une excursion au Spitzbergen, et Nicolas revient seul à Oslo, puis à Montréal où Éva Vos vient le rejoindre. Il écrit le scénario du film. Ce n'est qu'à la fin du roman qu'on aura à la fois le récit du passé de Sylvie et celui de sa mort. Ce n'est qu'à ce moment qu'on saura que Nicolas avait découvert l'histoire de Sylvie. Au.moment de la mort de sa mère, Sylvie prend son nom - Dubuque - et fait ainsi passer son père 
pour son amant. A Montréal, où elle vit avec Nicolas, elle continue de fréquenter son père. Mais ce passé, malgré les allusions faites ici et là, ne sera connu du lecteur qu'à la fin du roman (p. 224). Dans les pages qui suivent, on a le récit du meurtre de Sylvie par Nicolas. Et c'est le rapprochement de ces deux récits - dont l'exposition est retardée - qui provoquera la relation de cause à effet dans la compréhension du comportement de Nicolas.

Mais cette intrigue une fois connue, ce n'est qu'une partie du roman qui est comprise par le lecteur. II reste tout ce qui est mis entre parenthèsès dans le roman: Fortinbras, Hamlet, le temps et les diverses réflexions sur le sénario, sur le spectateur... et le revirement final: apparition du je du narrateur, et le film devient livre.

Celui qui s'est rendu jusqu'ici sait que ce n'est pas un film qu'il regarde par les yeux d'un spectateur, mais un livre qu'il continue luimême de feuilleter en tremblant. ( $N N$, p. 253)

On peut diviser le roman en deux grandes parties. Dans une première partie (du début à la page 150) on a le voyage de Montréal à Spitzberg et le retour de Nicolas à Montréal. La seconde partie se déroule entièrement à Montréal, où Nicolas écrit le scénario de son film. Mais, dans les pages 240 à 254, on peut voir une troisième partie, très brève, qui constituerait, selon une perspective habituelle dans les romans d'Aquin, la postface: Eva et Linda lisent le scénario, tout comme Suzanne, dans l'Antiphonaire, lisait le manuscrit de Christine. Et le revirement final du narrateur ressemble assez à l'attitude de RR récupérant le roman.

Avec Neige noire, on demeure dans l'univers romanesque: ce n'est pas un ciné-roman. II n'est que de le comparer brièvement à l'Immortelle ${ }^{5}$ de Robbe-Grillet pour voir immédiatement les différences: peu ou pas d'indications sur les personnages (vêtements, attitudes, positions précises), peu d'indications précises sur les plans ou les mouvements de la caméra. Quand il y en a, c'est plutôt pour dire l'effet qu'on doit rechercher, ou l'image qu'on doit obtenir et non la manière dont doit être placée la caméra. On ne peut pas dire cependant qu'il n'y a jamais de directives: on note des "fondus enchaînés", gros plans, flashes, superpositions, mais peu de remarques sur le cadrage des plans. II y a toute une partie de Neige noire qui serait difficile à rendre dans une adaptation cinématographique: les parenthèses où s'inscrivent les réflexions sur le scénario et les effets de temps qu'on doit rechercher. On reconnaît là le pararécit devrait-on dire para-scénario? - qui se distingue par sa mise entre parenthèses et que le narrateur lui-même appelle commentaire ${ }^{6}$.

II y a plusieurs niveaux dans Neige noire, et chacun traîne avec lui son mystère: l'espace géographique, l'espace culturel, par exemple, qui se confondent finalement dans le mythe de Undensacre. Tout cela s'inscrit dans le scénario du narrateur premier, dans lequel s'inscrit à son tour celui de Nicolas. Ce même Nicolas qui a joué le rôle de Fortinbras. Ce dernier personnage renvoie à la citation de Sören Kierkegaard en exergue du 
roman: "Je dois maintenant à la fois être et ne pas être ${ }^{7}$ " qui répond au dualisme de la question de Hamlet par un "double bind" où la conjonction ou fait place a la conjonction et. Ce qui importe dans Neige noire, ce n'est pas Hamlet, mais le personnage de Fortinbras, celui qui règnera sur le Danemark avant même de régner sur la Norvège. De même que ce qui reste de l'espace géographique, ce n'est pas la conjonction Natchez-Spitzberg, mais la recherche de Undensacre, lieu mythique de l'écriture. Même si c'est Nicolas qui a joué le rôle de Fortinbras pour la télévision, l'équation finale, faite par le narrateur, est celle de SylvieFortinbras:

personne ne sait si cet Undensacre n'est qu'une autre désignation d'Odense ou si cet Undensacre ne se situe pas plutôt dans le Jutland occidental où se rendait Gertrude pour retrouver secrètement Fortinbras, le jumeau d'Hamlet; un peu comme Sylvie Lewandowski se rendait parfois, mais jamais assez souvent, à Undensacre retrouver son père en secret. Personne ne connaît vraiment l'emplacement du tombeau de Fortinbras qu'Eva et Linda cherchent dans leur dérive amoureuse; personne non plus ne retrouvera celui de Sylvie [...] (NN, p. 250)

On retrouve dans Neige noire les effets de correspondance de l'Antiphonaire. La scène du meurtre de Sylvie (p. 234 à 240) à l'intérieur du refuge de Spitzberg a son répondant dans la scène de Linda ligotée dans l'appartement de Stan Parisé (p. 24 à 30). A la fin de cette dernière scène, d'ailleurs, la correspondance est visualisée - même si le lecteurspectateur n'en comprend pas encore la signification: "Plan filé de Linda ligotée sur le lit au cabin-cruiser Artic amarré non loin du mât de $\mathrm{Ny}$ Ålesund " (NN, p. 30).

Deux autres éléments provoquent des effets de correspondance à travers tout le roman: on' ne fait ici que les signaler. Les muscles du chagrin (p. $78,83,90,134,135,140,239,244$ ) et le théâtre illuminé sur lequel se clôt le roman (p. 118, 142-146, 227, 254). On retrouve aussi dans le roman, le paradigme viol(ence). Dans l'Antiphonaire, il y avait deux conjugaisons du paradigme : viol(ence) Renata/Zimara-Christine/pharmacien, d'une part, et meurtre Zimara/Antonella, Chigi/Antonella, JeanWilliam/pharmacien et Jean-William/Robert d'autre part. Dans Neige noire, viol et meurtre se conjuguent dans une même viol(ence) Nicolas/Sylvie. A la viol(ence) répond la conjugaison amoureuse Eva/Linda.

Si on reprend tous ces éléments, on obtient un synopsis qui éclairera la suite de l'analyse:

\section{SYNOPSIS}

1. MONTRÉAL (p. 7-32)

incident du pendentif

2. Départ (p. 32) voyage en AVION

(p. 38-45) (p. 32-50)

3. Oslo auto d'Eva Vos (p. 50-57)

Tromso Avion et Taxi (p. 57-60) 


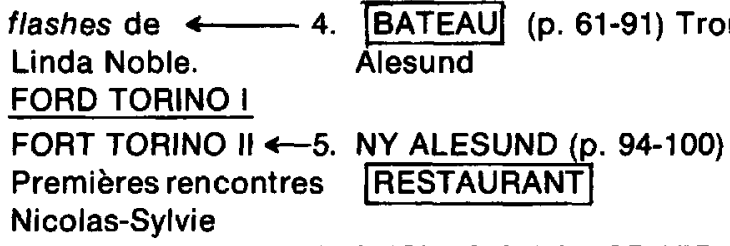

6. EXCURSION AU SPITZBERGEN (p. 101-114)

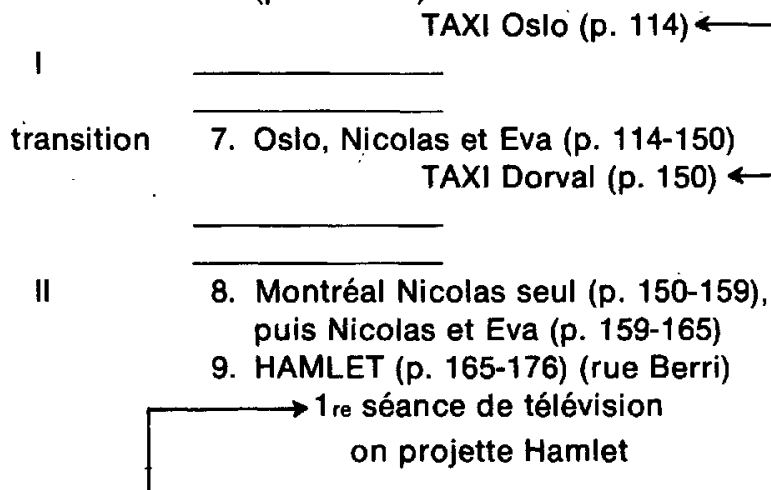

10. rencontre $M$. Lewandowskj-Eva (p. 177-181) retour d'Eva chez Nicolas

11. Hamlet II (p. 187-198) $\longrightarrow 2$ e séance de télévision on projette la scène du film "II était une fois dans l'Ouest", où Henry Fonda et Claudia Cardinale font l'amour.

12. essais de caméra (p. 199-200)

13. 2 e rencontre Michel Lewandowski et Eva au restaurant Bill Wong (p. 203-209)

Michel parle du meurtre de Sylvie par Nicolas. De retour chez Nicolas, Eva discute de cette rencontre.

rencontre de Sylvie et Michel tentative de suicide de Sylvie inversion

situation

Nicolas et Eva font l'amour

Eva et Nicolas discutent de Hamlet et du scénario.

15. Nicolas finit la rédaction du scénario (p. 221-227)

16. Eva et Nicolas discutent du scénario et rentonrent Linda Noble (p. 227-234) 


\section{SÉQUENCE DU MEURTRE DE SYLVIE (p. 234-240)}

UNDENSACRE

THULE

THÉATTRE

ILLUMINÉ

II convient de faire quelques remarques. L'espace géographique est ce qui permet le plus facilement de situer le roman. On voit l'importance du lieu où se déroule l'intrigue, et celle du déplacement d'un lieu à l'autre. Le voyage est l'occasion de flashes back révélateurs du passé des protagonistes. Ainsi, au voyage en avion de Montréal à Oslo, se superpose l'incident du pendentif; à la traversée en bateau de Tromso à Ny Älesund se superposent des flashes de Linda Noble et des événements de la Ford Torino à Montréal - le dévoilement est toujours progressif. Les moyens de transport sont des signifiants importants. Outre l'avion et le bateau, il y a les taxis. On a le départ de Dorval, à Montréal (p. 32), l'auto d'Eva Vos à l'arrivée à Oslo ( $p$. 50), puis le taxi, de l'aérogare au bateau, à Troms $\varnothing$ (p. 60). Après l'excursion au Spitzbergen, l'image du taxi à l'arrivée de Trondheim se superpose à l'image de l'hélicoptère cherchant le corps de Sylvie. Inversement, quand Nicolas est de retour à Dorval, le trajet en taxi est entrecoupé de flashes de l'hélicoptère.

On peut dire que le voyage dans le temps rejoint le voyage dans l'espace par la médiation de l'avion, du bateau ou de la voiture. Le déplacement spatial entraîne un déplacement temporel: le voyage est le lieu de la mémoire. On obtient, par surimpression, la composition de l'espacetemps (fictionnel). Un autre lieu important de la mémoire est le restaurant. C'est au restaurant de Ny Álesund que se révèle le passé des trois premières rencontres de Sylvie et Nicolas. Ces rencontres se superposent à celles de la Ford Torino, où le lecteur découvre progressivement qu'il s'agit bien de Sylvie dans cette scène de la voiture. C'est aussi au restaurant, à Montréal cette fois, que Michel Lewandowski et Eva se rencontreront à trois reprises (p. 177-181; p. 203-209; p. 244) pour parler de Sylvie ${ }^{8}$.

Un autre élément important, englobant le théâtre et répondant au scénario, est la télévision. On voit bien, dans le synopsis, l'inversion de situations lors des deux séances de télévision: l'une où Nicolas et Eva font l'amour durant la projection de Hamlet - pour laquelle Nicolas a joué le rôle de Fortinbras ( $N N$, p. 9-13 et p. 18-20) - et l'autre où les deux personnages discutent de Hamlet et du scénario de Nicolas, pendant que la télévision projette une scène amoureuse.

$\mathrm{Si}$ on essaie de regrouper ces éléments dans une problématique générale, on retrouvera la prépondérance de l'espace géographique. Ce 
dernier, dans Neige noire, se constitue de la même manière que le film: par surimpression ou superposition.

C'est encore au niveau du pararécit que se dévoile le fonctionnement du roman - le pararécit est, dans ce roman, le commentaire du narrateur qui fait partout transition entre les diverses scènes du scénario. Ce qui sert de révélateur, c'est le rêve de Nicolas, le «théâtre illuminén. Nicolas rêve qu'il joue Hamlet «dans une ville italienne située sur les bords de la mer de Barents...". II décrit cette ville "construite selon un plan concentrique " et parle du théâtre illuminé. Eva tente, en vain, de le convaincre qu'il a fait un cauchemar. Le commentaire du narrateur, entre parenthèses, poursuit cette discussion.

Après le cauchemar de Nicolas, le spectateur capitule et consent illico à rétrocéder au cinéaste l'archipel du Svalbard, ses villages de carton et ses glaciers fantastiques: il se peut d'ailleurs que, bien avant cette fracture, il ait décelé quelques ressemblances entre la dentelle glaciaire de I'Isfjorden et les frontispices invaginés du littoral de l'Alaska, entre les croupes du Cap Mitra et la chevelure basaltique des îles Sverdrup, entre la configuration du Kongstjorden et celle de Repulse Bay... Rien n'est plus corosif que le doute. L'enclave italienne conduit à la facticité du Spitzbergen qui mène, par induction, aux massifs crevassés de l'Alaska, plantés dans la mer de Barents par un cartographe délirant... (NN, p. 145-146)

\section{Et aussi :}

Inhabité, le décor devient insidieusement taxé d'irréalité et par un effet en miroir du cauchemar de Nicolas, ce décor devient transparent à tel point que son identité usurpée se laisse deviner, et qu'il n'est plus, pour les spectateurs prévenus, que l'envers d'un décor. Le Spitzbergen fond et dérive d'est en ouest sous la banquise soudée. $(N N$, p. 146)

L'envers du décor n'est pas sans rappeler "l'image renversée" des Alpes de Prochain Épisode. Et cette dérive d'est en ouest ouvre un univers de compréhension nouveau. Le théâtre illuminé, la gravure de Natchez ont la même fonction dans le scénario que celui de Nicolas au niveau du roman: révélateur par surimpression. On note que plusieurs scènes importantes se déroulent ainsi sur deux niveaux. On a vu déjà la composition de l'espace-temps par la superposition du voyage d'un lieu à l'autre et le souvenir du passé qui se dévoile progressivement. Le cinétisme du temps (passé $\rightarrow$ futur) est en surimpression par rapport à la progression (mouvement) géographique. On retrouve ce procédé de la surimpression dans la composition géographique. Ce qu'il faut comprendre, c'est que le récit de ce qui se déroule entre Nicolas et Sylvie en première partie (dans le synopsis, de 1 à 6 , et même 7 qui fait transition) a lieu en Norvège, à Oslo et Spitzberg. Le scénario donne le récit comme se déroulant en Norvège, mais le film doit être tourné à Repulse Bay et aux îles Sverdrup, du côté américain du pôle nord. (En page 247, Linda dira à Eva: «Nicolas Vanesse a nolisé un avion de Nordair et part demain pour Repulse Bay...") 
On a

d'un côté: le scénario

de l'autre: le tournage

Oslo

Montréal

Spitzbergen

Repulse Bay, Sverdrup

Entre les deux, on a une gravure de Natchez-under-the-Hill, ville de la Louisiane. Mais Natchez n'existe, dans le récit, que par une gravure suspendue au-dessus du lit de Michel Lewandowski, à Montréal, dans sa maison de l'île des Scurs. Natchez, c'est Montréal, tout comme Oslo est Montréal. On reste dans le cercle polaire, mais le Spitzbergen dérive d'est en ouest vers Sverdrup. On a donc Montréal (Oslo et Natchez) et les îles Sverdrup (Spitzbergen): entre les deux, le Groënland danois (Danemark/Norvège = Hamlet/Fortinbras) et Thulé, Undensacre ( l'île du Spitzbergen est une image retournée d'Undensakre”, $N N$, p. 250, dans le commentaire). Le lieu du scénario est èt n'est pas Spitzbergen... mais c'est le cercle polaire. Tout comme le scénario de Nicolas est et n'est pas biographique... Cette composition par surimpression se retrouve des grandes aux petites unités de l'œuvre. II sera plus facile d'en comprendre le fonctionnement après l'étude de quelques scènes du roman. Prenons en $14 \mathrm{du}$ synopsis, la séquence de repérage des lieux du tournage (NN, p. 210-220). On peut diviser la page selon la verticale.

Michel Lewandowski et Sylvie

Reperage des lieux par Nicolas

Nicolas et Eva prennent place dans la Ford Torino ( $N N$, p. 210) et sortent sur la rue Berri.

«Fondu enchaîné:"l'auto file sur de Maisonneuve vers l'Ouest."

“Coupe à la gare centrale, quai numéro 11.»

Michel Lewandowski à l'arrivée de la locomotive du New York Central. Sylvie monte en voiture avec lui.

“Plan filmé à l'intérieur de la maison de Michel Lewandowski" (NN, p. 213). Sylvie se dévêt partiellement et s'étend sur le.lit.
Nicolas prend des photos rue Drummond. En voiture, il continue avec Eva jusqu'au terrain de l'administration du Port de Montréal, en bordure du fleuve, “là même où se situe la scène entre Nicolas et Sylvie quand elle lui demande de rouler toute la nuit jusqu'à Natchez-under-the-Hill » (NN, p. 212). 
"La grande chambre à coucher. Gros plan de Sylvie qui vient de donner un long baiser à Michel Lewandowski » (NN, p. 214). Gravure de Natchez. Sylvie appelle Michel «papa».

(commentaire du narrateur: Michel est le père de Sylvie)

Sylvie dit à son père que la situation

ne peut plus continuer. «double fellatio "-gravure de Natchez. Coupure (NN, p. 216)

"La chambre à coucher dans la maison de Michel Lewandowski » (NN, p. 216). Sylvie dit à son père: "C'est la dernière fois, il le faut..." Sylvie et Michel font l'amour. maison qu'il a repérée.
"Coupe franche à la Ford Torino". A l'île des Sœurs, Nicolas repère un immeuble et monte au dernier étage de la tour prendre des photos. Au moment où il «place l'œil dans le viseur» de sa caméra, on voit l'image d'une maison à pignons, puis le foyer se fait sur le plan d'une fenêtre où on aperçoit une silhouette.

Coupure.

Nicolas rejoint Eva et laisse là la voiture pour aller voir de plus près la

"Coupe à l'extérieur. Nicolas, un genou par terre, prend des photos de repérage». (NN, p. 219)

“Montage de plans presque allusifs de Sylvie Lewandowski et de son père, dans le lit; plans intercalaires de Nicolas encerclant la maison de Michel Lewandowski dans un filet de cadrages fixes. $(N N$, p. 219)

"Intérieur de la maison". (commentaire du narrateur)

On distingue de moins en moins le couple.

"Plongée faite de l'immeuble" d'où l'on voit la maison de Lewandowski, Nicolas qui la contourne et qui revient vers Eva. Les deux s'éloignent de la maison ». (NN, p. 220)

(commentaire du narrateur)

Puis on passe à l'appartement de la rue Berri. 
On voit bien comment deux séquences sont développées simultanément. Le commentaire du narrateur va dans ce sens:

Nicolas a donc repéré une maison fictive qu'il veut utiliser comme étant la vraie maison de Michel Lewandowski et, par une certaine ironie du sort, cette maison est vraiment la demeure de Michel Lewandowski. Toutefois, une fausse simultanéité est suggérée au spectateur entre la scène qui se déroule dans la chambre des maîtres et l'opération du repérage [...] La préparation du film se déroule, somme toute, au niveau de la fiction (car ce qui n'est pas connu n'est-il pas fictif?) tandis que let scénario lui-même est échafaudé sur la vérité. Et ce qui s'est déroulé à l'île des Sœurs est une combinaison nouvelle du motif vérité-fiction: la fiction, plus large que la vérité, parce qu'indéterminée, englobe accidentellement la vérité. ( $N N$, p. 220)

On retrouve le fonctionnement de cette séquence dans tout le roman. On a partout deux éléments qui se superposent.

Cette composition de l'espace géographique par surimpression rejoint la problématique de l'écriture. La fonction de l'espace culturel dans l'écriture est en rapport homologique avec la fonction de l'espace géographique du récit dans la narration. L'espace de l'écriture du roman est en surimpression par rapport à l'espace culturel - d'un moment de l'histoire le tout forme l'univers romanesque.

\section{FICTION}
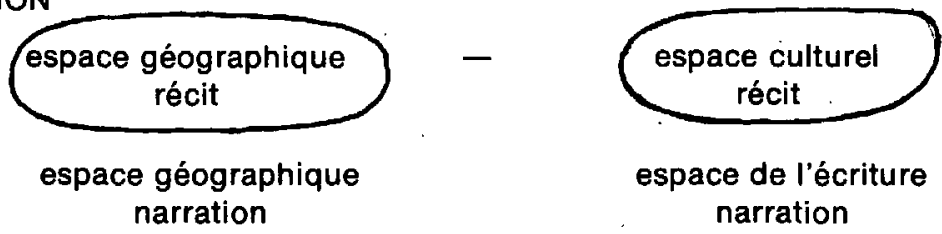

On pourrait, à partir de ce schéma, reprendre les quatre romans.

Prochain Épisode

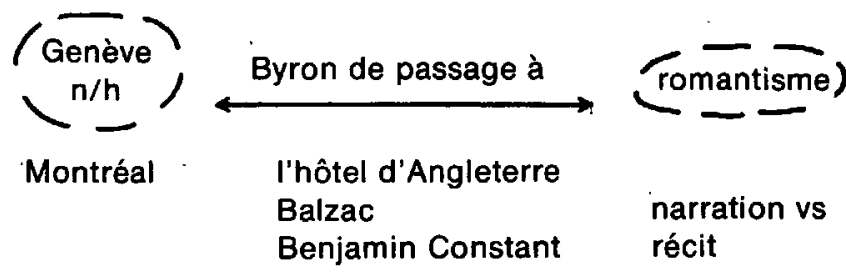

L'Antiphonaire

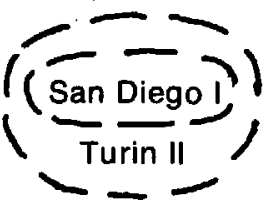

Montréal

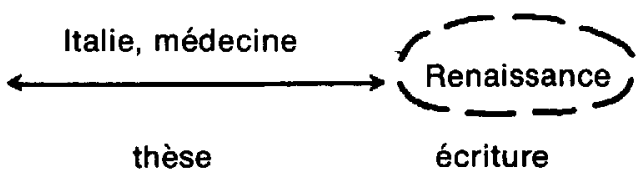


Trou de mémoire
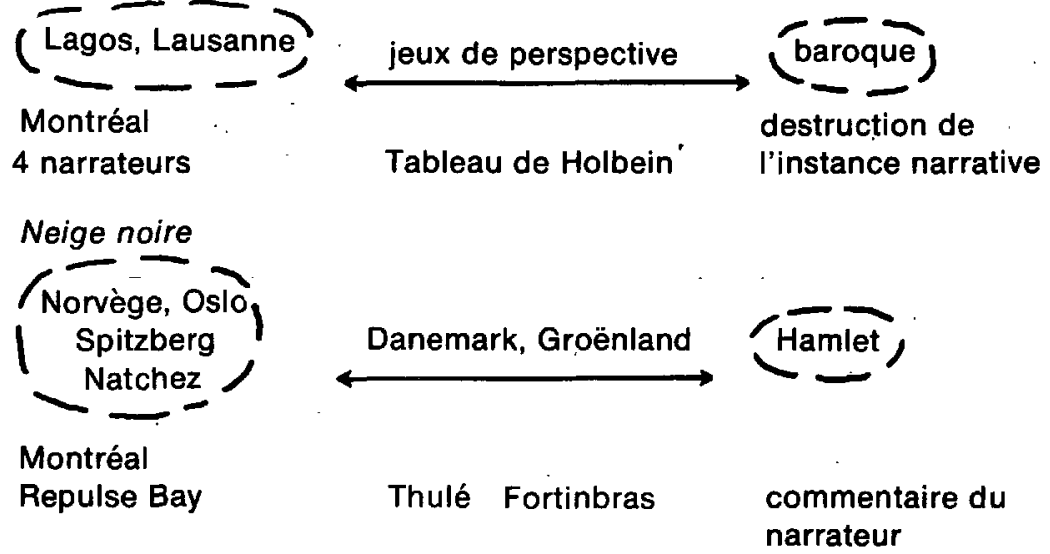

L'espace géographique se rattache à l'espace narratif par la médiation du récit. Le lieu du récit n'est pas toujours le même que celui de la narration:

\begin{tabular}{|c|c|c|c|}
\hline Prochain Épisode & $\begin{array}{l}\text { récit } \\
\text { château } \\
\text { d'Echandens }\end{array}$ & $\begin{array}{l}\text { narration } \\
\text { clinique } \\
\text { prison }\end{array}$ & $\begin{array}{l}\text { Le roman a en fait } \\
\text { été écrit à Montréal. }\end{array}$ \\
\hline L'Antiphonaire & $\begin{array}{l}\text { Turin, } \\
\text { San Diego }\end{array}$ & $\begin{array}{l}\text { (San Diego) } \\
\text { Montréal }\end{array}$ & \\
\hline Trou de mémoire & $\begin{array}{l}\text { Montréal } \\
\text { Lagos } \\
\text { Lausanne }\end{array}$ & Montréal & \\
\hline Neige noire & $\begin{array}{l}\text { Oslo } \\
\text { Spitzberg }\end{array}$ & Montréal & $\begin{array}{l}\text { narrateur premier: } \\
\text { C'est à Montréal } \\
\text { qu'on voit Nicolas } \\
\text { écrire son scénario. }\end{array}$ \\
\hline
\end{tabular}

La liaison entre l'espace géographique et l'espace culturel se fait dans le récit, soit par un personnage, soit par un lieu géographique, soit par un processus d'écriture. Cette écriture est liée à son tour à l'espace culturel par le pararécit - ou les commentaires. La relation écriture-narration se fait par la médiation du récit puisque cette relation est redevable des rapports précédents. II est donc impossible d'exclure le récit de la fiction. S'il y a refus du récit traditionnel, il n'y a donc d'autre voie pour le récit que celle du "double bind". C'est ce vers quoi tend le scénario.

Dans Neige noire; on cherche une nouvelle définition de la fiction. C'est la préoccupation de Nicolas quand il parle d'autobiographie: il 
cherche à éclairer les rapports réalité-fiction. Tout comme son scénario, Nicolas est ouvert à tous les possibles du récit: les relations réalité-fiction sont dépassées.

Dans mon scénario, la fiction n'est pas un piège, c'est elle, plutôt qui est piégée par une réalité qu'elle ne contenait pas et quil l'envahit hypocritement... (NN, p. 147)

Linda Noble non plus ne saura plus s'y retrouver:

II n'est plus question, pour Linda, de démêler la réalité de la fiction puisque la fiction est inextricablement mêlée aux mailles de la réalité et qu'en dissociant l'une de l'autre, Linda ne saurait plus, en fin de compte, si c'est la fiction qu'elle isole ou ce qu'il est convenu de désigner comme la réalité qui est contaminée par une fiction encore plus frissonnante. (NN, p. 243)

Ce problème des rapports réalité-fiction prend toute son importance dans la discussion du meurtre de Sylvie; Nicolas a-t-il vraiment tué Sylvie?

Dans la première partie du roman, il y a une coupure après l'excursion au Spitzbergen: on voit Nicolas revenir seul, puis faire les recherches avec I'hélicoptère norvégien. De retour à Oslo, il rencontre Eva et lui dit que Sylvie est morte accidentellement d'une chute dans un précipice. Devant le silence d'Eva, il lui jure qu'il ne l'a pas tuée, mais que Sylvie s'est suicidée. Plus tard, quand Nicolas écrira son scénario, Eva lui demandera toujours comment Sylvie doit mourir dans le scénario ou dans le film.

Si on excepte la rencontre d'Eva et de Nicolas à Oslo (NN, p. 123-129), le premier à parler du meurtre de Sylvie pour le scénario sera Michel Lewandowski.

La meilleure solution serait toutefois le meurtre... Le voyage de noces interrompu par un meurtre... Le couple est détruit au moment même où il se formait dans l'intimité... (NN, p. 207)

Le commentaire du narrateur - qui suit cette déclaration - abonde dans le même sens:

Le meurtre vaut mieux que le suicide, car il dévoile une intention pure de destruction. Tuer pendant le vovage de noces. c'est le renversement le plus bouleversant de l'amour. (NN, p. 207)

Quand Eva rapporte le propos de Michel Lewandowski à Nicolas, elle lui demande ce qu'il pense de l'idée du meurtre pour son scénario. Il lui répond:

D'emblée c'est non, parce que je veux pas [sic], au terme du scénario, déboucher sur la fiction... (NN, p. 210)

Un peu plus tard, Nicolas dira:

Je suis bien préoccupé par tout ce qui me reste à faire dans le scénario... Et cette idée du meurtre final de Sylvie me tourmente. Je me dis que Michel Lewandowski a peut-être raison sur le plan formel... [...] mais cela bouleverserait trop mon scénario que j'ai 
construit à partir de la vérité... Je vois encore Sylvie courant à toute allure vers le précipice, et moi, comme un infirme, ne trouvant rien de mieux que de crier pour essayer de la rattraper... Chose certaine, je ne réussirai jamais à imaginer que je la pousse dans le dos... (NN, p. 212)

Cette version du suicide est corroborée par le récit d'une précédente tentative de Sylvie à l'hôtel Bonaventure (NN, p. 226), confirmé semble-t-il par Michel Lewandowski (NN, p. 246).

Après le repérage des lieux du tournage, Nicolas entreprend de terminer son scénario. II dit à Eva:

Au fait, j'ai choisi la solution du meurtre. J'ai résolu, finalement, de tuer Sylvie. Tu ne trouves pas cela étrange qu'après avoir commencé sur le mode autobiographique, je finisse ce scénario en masquant la vérité...? (NN, p. 222)

Le narrateur poursuit, dans son commentaire:

Le suspense porte sur le processus du meurtre, non pas sur sa possible effectuation. Nicolas d'ailleurs a envisagé de la tuer en la poussant dans le précipice. Mais quelle que soit la méthode employée, il reste qu'il faut trouver une concaténation homicide qui tiendra lieu de justification du dernier moment. Odi et amo...? Est-ce possible de pousser jusqu'au meurtre la virulence catullienne? Peut-être, après tout, quand on se rappelle de quelle façon il a été trahi par cette folle dont il était fou... (NN, p. 223-224)

Mais cette discussion, concerne-t-elle la réalité ou la fiction? Quand Nicolas a fini la rédaction de son scénario, Eva, profitant de ce qu'il dort encore, s'empare du manuscrit et lit avec stupéfaction:

Sylvie mourra comme elle est morte; ne rien changer, ne rien modifier. Les gestes doivent être accomplis avec lenteur et jusqu'au bout, y compris... (NN, p. 228)

Eva remet le manuscrit sans rien dire. Lorsque Nicolas s'éveille, ils partent pour Montréal où ils doivent rencontrer Linda Noble. Eva lui demande alors s'il a tué Sylvie, dans son scénario. Contredisant ce qu'on vient de lire quelques lignes plus haut, Nicolas répond:

J'ai passé la nuit à chercher une façon de la tuer... Je n'arrive à rien de bon et je crois, en fin de compte, que je vais laisser le meurtre en blanc dans le scénario... D'ici le tournage, j'aurai bien le temps de concevoir cette séquence. (NN, p. 229)

Plus tard, Eva oblige Nicolas à lui dire que, dans le scénario, il poignarde Sylvie et la jette dans le précipice ( $N N$, p. 232). Après un commentaire du narrateur sur l'utilisation des scènes érotiques au cinéma et sa correspondance avec l'art du nu au temps de la Renaissance, on a la séquence du meurtre de Sylvie ( $N N$, p. 234-240). On passe ensuite à la lecture du scénario par Lịnda et Eva, puis à sa discussion. Eva, par tous ses arguments, convaincra Linda de la véracité du meurtre et de la manducation de Sylvie par Nicolas. Mais le lecteur restera partagé, ébranlé par la discussion réalité-fiction: Nicolas a-t-il ou non tué Sylvie? Le roman délaissera 
ces problèmes pour se terminer dans une apocalypse amoureuse. Mais cette brouille des rapports réalité-fiction rejoint, par-delà le récit, les relations scénario-récit-commentaire. II faut revenir au narrateur de Neige noire, à son commentaire et à son scénario. Ce qui s'inscrit dans les parenthèses du roman est en fait le commentaire du narrateur qui englobe le scénario et le scénario de Nicolas qui s'inscrit à son tour dans celui du narrateur premier:

le scénario qu'il lle lecteurl a lu depuis le début se trouve enchâssé dans le présent commentaire et [...] ce commentaire explique la publication de ce qu'il enchâsse et reproduit, structuralement, le stratagème de la pièce dans la pièce, mais à l'envers: ce n'est pas une insertion du plus petit dans le plus grand, mais du plus grand dans le plus petit [...] (NN, p. 195)

Le narrateur poursuit:

Le scénario fait penser à une contre-réponse; il est inséparable du sujet qui l'encadre et du contre-sujet qui se greffe à lui, tout autant qu'il se nourrit de la réponse dont il émane. (NN, p. 196)

La fiction de Neige noire est donc une équation à trois termes: le commentaire (sujet qui encadre), le scénario (contre-réponse) et le scénario de Nicolas (contre-sujet qui s'y greffe). Selon une glose familière chez Aquin, les relations entre les éléments s'inversent et c'est le commentaire qui englobe tout. On retrouve l'importance habituelle du pararécit qui amène tout le roman à lui. Cependant, le plus intéressant est la superposition scénario/récit qui produit un effet nouveau. En effet, le scénario de Nicolas tend à paraître comme le récit du scénario fait par le narrateur premier. Avant de poser la question du scénario - à quoi correspond-il, quelle est sa fonction? - il faut reprendre les termes de la fiction et voir leur évolution dans les romans.

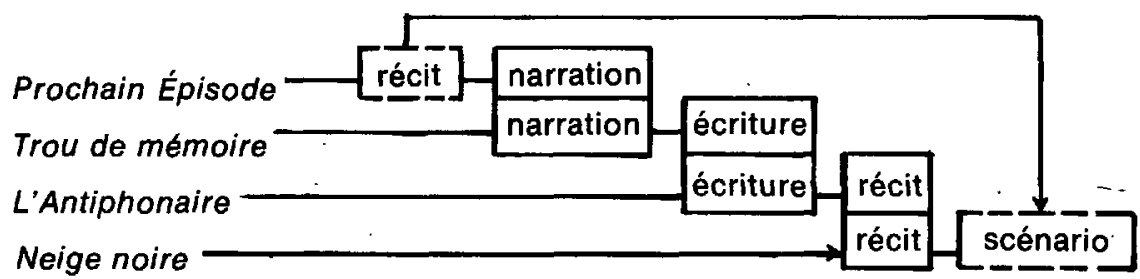

D'un roman à l'autre, une même recherche se poursuit. De l'un à l'autre, un terme de la fiction sert de transition. Ainsi, dans Trou de mémoire, on conserve un terme du roman précédent, la narration, pour le confronter à des problèmes d'écriture. On retrouve la triple relation narration-écriture-récit, où l'écriture fait le relais entre la narration et le récit. Neige noire reprend finalement la problématique du dernier roman et revient à la question du récit. Ce qui était à l'état latent à la fin de Prochain Épisode - refus du récit, ébauche d'un scénario comme récit au futur antérieur - se matérialise dans le dernier roman, avec le scénario. L'apparition de ce dernier terme est donc l'aboutissement d'une recherche de l'écriture, et pas seulement un hasard de l'aventure romanesque. 
Le scénario répond à deux impératifs de l'écriture d'Aquin: 1. le jeu des correspondances tel qu'on le voit fonctionner dans l'Antiphoriaire, par exemple, non seulement dans la modulation des thèmes, mais dans les techniques d'articulation des séquences et des chapitres (la grammaire des thèmes); la technique de la surimpression - images superposées du film - que peut exploiter le scénario répond à ce jeu des combinatoires de l'écriture d'Aquin. 2. l'importance du temps: l'implacable déroulement du temps est omniprésent dans tous les romans (que ce soit dans la structure ou la construction- de la distance-narrative, ou-dans les thèmes et objets qui sont partout dans le texte). Le narrateur dit lui-même en quoi le film rend bien cette obsession du temps:

Le film, mieux que tout autre moyen d'expression, rend bien cette fluidité achérontique, dans la mesure au moins où il ne s'arrête jamais avant la fin de sa propre projection: ( $N N$, p. 149)

Le scénario répond à certaines exigences fondamentales de l'écriture d'Aquin. Mais le récit n'est pas exclu, et on n'a pas adopté exclusivement le scénario. L'intérêt de Neige noire vient justement de cette utilisation du scénario sur fond de récit, accompagné d'un commentaire, pararécit, qui partout fait transition, appuie ou souligne tel ou tel aspect du scénario. C'est ici que l'on retrouve la citation "être et ne pas être», dans ce récit et non-récit qu'est le scénario. Considéré dans sa potentialisation de récit, le scénario correspond mieux aux «possibilités» multiples de l'écriture et de son discours. Cette façon d'utiliser un scénario/récit rejoint la superposition comme fonctionnement global du roman - dans la composition des espaces géographique, culturel, narratif, scriptural, etc. II y a donc une cohérence de fonctionnement romanesque, même si la fiction est affectée d'un coefficient inconnu. II reste à analyser le paradigme viol(ence), qu'on a déjà vu dans les romans précédents, mais dont la «lecture "est éclaircie par celle de Neige noire ${ }^{9}$.

Il y a deux moments, dans le roman, où la distance entre Nicolas et le narrateur tend vers zéro: à la fin de la rédaction du scénario ( $N N$, p. 222-227) et à la suite d'un rêve de Nicolas (NN, p. 155-159). Ce dernier rêve a une fonction aussi révélatrice que celle du rêve du théâtre illuminé (NN, p. 142-144). Dans cette séquence, Nicolas fait un rêve qui condense la chute de Sylvie au Spitzbergen et l'incident du pendentif. Puis, réveillé,

II se relève complètement, se rend à sa table de travail. II ouvre le cahier noir qui est sur le sous-main et se met à écrire sans s'interrompre.

Suit alors le texte de Nicolas, qui se termine par ces mots: “Ce texte même que je m'apprête à terminer, fait partie du scénario" (NN, p. 157). Mais dans le commentaire qui suit immédiatement - entre parenthèses -, le narrateur précise:

Tout au long de ce texte écrit, garder la caméra fixée sur Nicolas; déconcerter en quelque sorte le spectateur qui attend des flashes intercalaires ou des métonymies visuelles du texte écrit à la main par Nicolas. Cela serait précisément trop facile. (NN, p. 157) 
On relève une contradiction: le lecteur connaît le texte de Nicolas, mais le spectateur, lui, ne le connaît pas. On voit que le scénario de Nicolas ne recouvre pas tout le roman, mais que ce dernier le déborde - on recoupe les réflexions du narrateur premier dans son commentaire de la page 197. Le scénario, dont on prend lecture et sur lequel se greffe le commentaire, n'est pas celui de Nicolas, mais celui du narrateur du roman ${ }^{10}$. Nicolas veut que son texte soit intégré au scénario, mais le narrateur précise que le spectateur ne connaîtra pas ce texte - contrairement à la séquence 222-227, où on aura des flashes du texte écrit par Nicolas. Mais poursuivons la lecture du commentaire.

Le présent employé depuis le début, est le temps du scénario parce qu'il ne rapporte rien mais transmet une intention - et pas n'importe quelle intention: celle d'un rapt! Le récit d'un viol imagé est d'une certaine fadeur si on le compare au projet de viol; l'intention projetée transcende toujours son passé défini. Cela va de soi; mais ce qui coule moins d'évidence, c'est la relation qui existe entre cette terminologie violente et la situation du spectateur du film. (NN, p. 158).

Le développement qui suit cet extrait - et que l'on trouve reproduit à l'endos du livre - démontre que le spectateur est violé par le film qu'il regarde et qui le "pénètre hypocritement". On note d'abord, dans la citation, l'opposition qui est faite du récit et du scénario, et la suprématie de ce dernier comme projet et force persuasive. C'est en quelque sorte la justification de son choix du scénario que fait ici le narrateur. Cette réflexion fait comprendre la fonction du paradigme viol(ence) dans l'œuvre romanesque.

Le paradigme viol(ence) - on le laisse entendre - se rattache au spectateur... et au lecteur, donc à la lecture. On peut l'entendre dans deux sens: 1 . le lecteur lit le récit d'un viol et 2 . le lecteur est violé (envahi) par la lecture qu'il fait. L'écriture est une intention de viol(ence) vis-à-vis du lecteur ${ }^{11}$; mais pour être actualisée, cette intention a nécessairement besoin de la lecture. L'écriture intègre donc la lecture à son activité puisqu'elle la présuppose au moment où le livre se fait. Le motif ou "thème" de viol(ence) dans le récit qu'on lit répond donc à la viol(ence) qui est faite au lecteur agressé. Mais cet état du lecteur, on le retrouve déjà reflété dans le corpus du texte. Dans l'Antiphonaire, la postface montre Suzanne qui lit le manuscrit de Christine. C'est là un acte important - qui entraîne la mort d'Albert Franconi. Dans Trou de mémoire, ce processus de lecture est constant - on le voit dans les notes et dans la succession des narrateurs. Chaque narrateur qui écrit a lu le texte de son prédécesseur. L'éditeur a lu le texte de P. X. Magnant, le corrige et écrit à son tour. RR lit le récit de P. X. Magnant, celui de l'éditeur, et écrit à son tour pour réfuter les affirmations de l'éditeur, qui après lecture du texte de RR, écrira à son tour pour contredire les réflexions de RR. Ce roman est fait du processus écriture-lecture-écriture, etc., qui s'engendre à l'infini. C'est d'ailleurs ce processus qui amène la destruction de l'instance narrative. 
Dans Neige noire, on a la même situation. Eva et Linda lisent le scénario, en discutent. Tout comme Suzanne dans l'Antiphonaire, c'est Eva qui informera le lecteur du destin final des personnages. Mais contrairement à Suzanne, la lecture du scénario n'entraînera pas la mort suicide ou meurtre - mais l'amour entre les deux lectrices. Cet épisode pourrait s'intituler: «le salut amoureux par la lecture...».

On voit que les débuts et fins de romans, chez Aquin, ne sont pas simplement des artifices de composition, mais une nécessité inhérente à sa problématique de l'écriture. La thématique de la viol(ence) et du meurtre, et de leur corollaire, l'érotisme, trouvent leur fondement dans cette problématique, écriture-viol(ence)-lecture. Car il ne peut y avoir de synthèse possible de termes contradictoires que dans la viol(ence); cette synthèse dialectique - provisoire - se retrouve dans le soleil de la nuit polaire:

Ce n'est pas une nuit blanche, mais une nuit ensoleillée, bel et bien ensoleillée, pénétrée de toutes parts par un intrus, violée par la lumière, réalisant ainsi le mariage du jour et de la nuit. (NN, p. 65)

On retrouve l'itinéraire vers le pôle nord, cette recherche d'Undensacre comme le lieu extrême où se produit, dans le processus dialectique, cette harmonie des contraires qu'on voudrait voir perdurer...

Christiane Houde

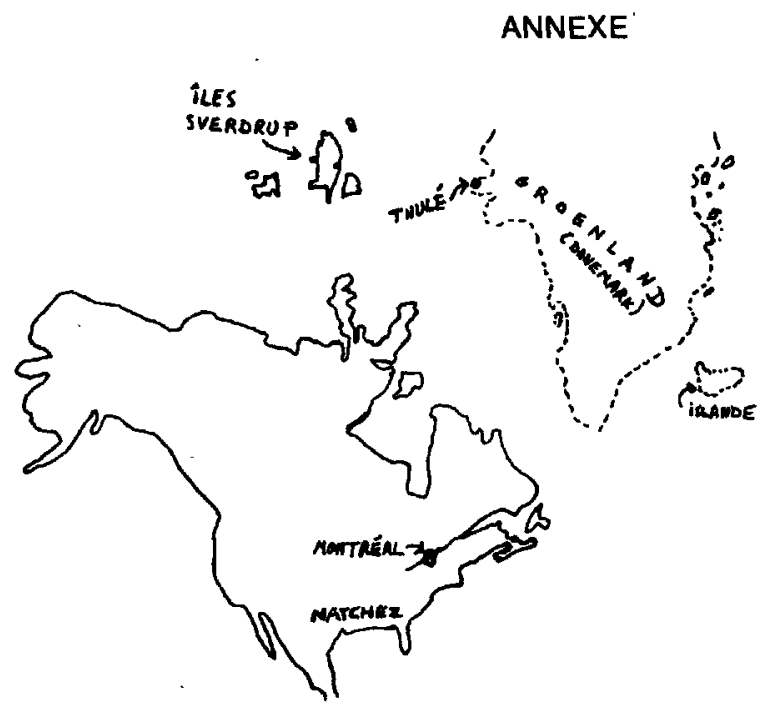

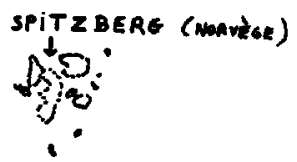

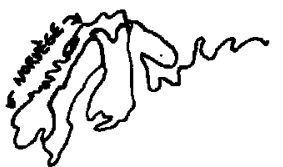


1. Montréal, La Presse, 1974. Notre abréviation: NN.

2. On retrouve l'expression "neige noire» dans le scénario, en page 197, lors de la projection à la télévision du film "ll était une fois dans l'Ouest".

3. Les Cantons de l'Est sont le lieu de prédilection des amants dans l'espace géographique aquinien...

4. Voir la carte donnée en annexe.

5. Paris, Minuit, 1963.

6. De "para", par-delà le récit. II est discours sur le récit: l'écriture se prend pour objet de son propre discours. Ce pararécit est présent à tous les niveaux de la fiction romanesque.

7. Il faut garder la tension des contradictoires. On verra ici l'allégorie de ce roman, où le récit est et $n$ 'est pas tout à la fois.

8. Doit-on relier l'importance de ce lieu-restaurant à la scène de la manducation de Sylvie par Nicolas?... II faut noter, aussi, l'étrange ressemblance entre la rencontre Lewandowski-Eva (en p. 203-209) et celle de Nicolas et Eva à Oslo, où il est question du meurtre de Sylvie (NN, p. 123-129).

9. Ce terme permet de rendre compte à la fois du viol physique et de la violence faite à autrui, de l'agression psychique et du meurtre.

10. Si on se permet d'insister sur ce point, c'est que la chose n'a pas semblé évidente pour tous - tout comme le roman dans le roman dans l'Antiphonaire. Ainsi, Jacques Pelletier dans son article "Sur Neige noire - L'CEuvre ouverte de Hubert Aquin ", en parlant de la fin du roman oú Eva et Linda font la lecture du scénario de Nicolas, écrit: "Notons que cette "conclusion" figure aussi dans le scénario de Nicolas, ce qui ne va pas sans laisser songeur... " In Voix et images, vol. I, $n^{\circ} 1$, septembre 1975, p. 23.

11. Cette affirmation n'est pas trop "viol(ente)" si on se réfère à l'entrevue accordée par Aquin (Voix et images, vol. I, $n^{\circ} 1$, septembre 1975, p. 9-10) où ce dernier parle de «son agressivité foncière" vis-à-vis du lecteur considéré comme celui qui pose "le problème de l'altérité dans toute son amplitude". Quand on lui dit qu'il y a là un «rapport sado-masochiste", il répond: "J'accepte bien volontiers. Je me venge du lecteur, je l'admets. C'est une relation bien ambivalente. Je me réjouis de sa lecture mais je suis méchant pour lui à l'instant même où je suis conscient qu'il me réjouit de sa lecture. » 